\title{
Kontrastmittelsonografie zur Charakterisierung von Lebertumoren: Praktische Aspekte
}

\section{Contrast-enhanced ultrasound for characterisation of focal liver lesions, practical advice}

Autoren

Institute
E. Fröhlich ${ }^{1}$, C. Jenssen ${ }^{2}$, A. Schuler ${ }^{3}$, C. F. Dietrich ${ }^{4}$

Medizinische Klinik, Karl-Olga-Krankenhaus, Stuttgart, Germany

2 Medizinische Klinik, Krankenhaus Märkisch Oderland GmbH, Wriezen, Germany

Medizinische Klinik, Helfenstein Klinik, Geislingen, Germany

Medizinische Klinik, Caritas-Krankenhaus Bad Mergentheim, Germany
Schlüsselwörter

- Kontrastverstärkte Sonografie

- Lebertumoren

- fokal noduläre Hyperplasie

- Hämangiom

- Leberzelladenom

- hepatozelluläres Karzinom

Key words

- contrast-enhanced ultrasound

- liver tumors

- focal nodular hyperplasia

- hemangioma

- liver adenoma

- hepatocellular carcinoma

eingereicht 28.12 .2014

akzeptiert $\quad 6.7 .2015$

\section{Bibliografie}

Dol http://dx.doi.org/ 10.1055/s-0035-1553491

Z Gastroenterol 2015; 53 :

1099-1107 @ Georg Thieme Verlag KG Stuttgart - New York . ISSN 0044-2771

\section{Korrespondenzadresse} Prof. Christoph F. Dietrich Innere Medizin 2, CaritasKrankenhaus Bad Mergentheim Uhlandstr. 7

97980 Bad Mergentheim Germany

Tel.: ++49/0 7931/5822 01

Fax: ++ 49/0 7931/582290

christoph.dietrich@ckbm.de

\section{Zusammenfassung \\ $\nabla$}

In diesem Übersichtsartikel werden evidenzbasierte und praktische Aspekte zur Kontrastmittelsonografie von Leberraumforderungen dargestellt und kritisch bewertet. Neben den im Vergleich zur B-Bild-Sonografie verbesserten Möglichkeiten der Detektion und Charakterisierung werden Limitationen und Fehlermöglichkeiten dargestellt und ein Vergleich zu radiologischen Schnittbildverfahren gezogen. Behandelt werden auch Empfehlungen zur Wahl des Ultraschallgeräts und des Kontrastmittels sowie notwendige Qualifikationen des Untersuchers für eine sichere Befundinterpretation.

\section{Einleitung}

\section{$\nabla$}

Die Kontrastmittelsonografie (contrast-enhanced ultrasound „CEUS“, „echosignalverstärkte Sonografie“) hat sich zur verbesserten Detektion von Lebermetastasen [1] und zur Lebertumorcharakterisierung etabliert [2,3]. Leitlinien zum kontrastverstärkten Ultraschall wurden erstmals von der European Federation of Societies for Ultrasound in Medicine and Biology (EFSUMB) 2004 veröffentlicht. Aktualisierte EFSUMB-Leitlinien wurden 2008 [4], 2011 [5] und 2012 publiziert [6-8] sowie kommentiert [9-12]. Die DEGUMVeröffentlichungen bestätigen die unizentrisch erhobenen Ergebnisse multizentrisch [3, 13 -17]. CEUS gilt als ein Verfahren mit höherer Ortsauflösung und Echtzeitdarstellung (real time) gegenüber den kontrastverstärkten bildgebenden Verfahren Computertomografie (CT) und Magnetresonanztomografie (MRT). Dennoch hat sich CEUS bisher nicht der diagnostischen Bedeutung und Kosteneffektivität der Methode entsprechend als ubiquitär breit eingesetzte Diagnostik neben CT und MRT durchsetzen können $[18,19]$. Das liegt an der noch nicht flächendeckend vorhandenen Untersucherqualifikation sowie daran,

\section{Abstract \\ $\nabla$}

This review article reports on evidence-based and clinical aspects of contrast-enhanced ultrasound of liver masses. The advanced possibilities of contrast-enhanced ultrasound to detect and characterise liver tumors are described as well as limitations and pitfalls of the method. The choice of the ultrasound device and contrast agents as well as the qualifications of the examiners according to guidelines are discussed.

dass für eine reproduzierbare optimale Ergebnisqualität des Leber-CEUS die Abläufe dieser aussagekräftigen Untersuchungsmethode nicht ausreichend standardisiert sind, aber auch an der unzureichenden Kostenerstattung von CEUS im ambulanten Setting (in der stationären Behandlung ist CEUS über OPS abgebildet). Auf die Zulassungsproblematik wird hier nicht weiter eingegangen und auf die Literatur verwiesen [20 - 24]. Diese Übersichtsarbeit dient der Standardisierung und Qualitätssicherung und geht folgenden Fragen nach:

1. Was sind die Voraussetzungen für CEUS der Leber?

2. Wer ist für CEUS der Leber qualifiziert?

3. Wann sollte CEUS der Leber (anstatt CT oder MRT) erfolgen?

\section{Voraussetzungen \\ $\nabla$}

Welches Kontrastmittel?

Schallverstärkung bei der KM-Sonografie wird durch Resonanzeffekte kleinster Gasbläschen in den Blutgefäßen hervorgerufen. Seit 1991 sind standardisierte Ultraschallkontrastmittel für die 
Rechtsherzdiagnostik (Echovist ${ }^{\circledR}$ ) und seit 1995 lungengängige Ultraschallkontrastmittel (Levovist ${ }^{\circledR}$ ) kommerziell erhältlich. Bei dieser ersten Generation von Ultraschallkontrastmitteln (UKM) wurden Luftbläschen mit einer Albumin-Hülle stabilisiert (Albunex $^{\circledR}$, Echovist ${ }^{\circledR}$, Levovist ${ }^{\circledR}$ ).

Seit 2001 sind Kontrastmittel der 2. Generation mit schwer wasserlöslichen Gasen auf dem Markt (SonoVue ${ }^{\circledR}$, Optison ${ }^{\circledR}$, Sonazoid $^{\circledR}$ ), die eine deutlich erhöhte Stabilität und Kontrastdauer aufweisen und somit für eine kontinuierliche Untersuchung geeignet sind. UKM mit gewebespezifischer Affinität (Sonazoid ${ }^{\circledR}$ ) lagern sich im RES der Leber und Milz an. Einige Produkte (Albunex $^{\circledR}$ ) werden inzwischen nicht mehr angeboten, da sie durch besser wirksame Präparate ersetzt wurden. Aktuell wird Optison wieder angeboten [3, 13-17].

In Deutschland sind 2 Kontrastmittel für die echosignalverstärkte Untersuchung der Leber zugelassen. Das Kontrastmittel der 2. Generation $\left(\right.$ SonoVue ${ }^{\circledR}$ ) gilt in Europa heutzutage als Echosignalverstärker der ersten Wahl $[25,26]$. Levovist ${ }^{\circledR}$ wurde noch lange Zeit insbesondere zur Abklärung von kleinen Raumforderungen $<30 \mathrm{~mm}$ in der zirrhotisch umgebauten Leber sowie in der Diagnostik vergrößerter abdomineller Lymphknoten mit ausreichend hoher Dosierung $(4 \mathrm{~g})$ und Konzentration $(400 \mathrm{mg} / \mathrm{ml})$ eingesetzt $[2,27,28]$. Sonazoid ${ }^{\circledR}$, das sich stärker noch als Levovist $^{\circledR}$ durch Speicherung im retikuloendothelialen System und damit eine leberspezifische Spätphase („Kupffer-Phase“) auszeichnet, ist in Europa bisher nur in Norwegen und Dänemark verfügbar. Japanische Arbeiten berichten über ausgezeichnete Ergebnisse insbesondere in der Diagnostik des hepatozellulären Karzinoms und anderer Leberraumforderungen $[29,29]$.

\section{Welches Gerät?}

Alle Gerätehersteller hochwertiger Ultraschallgeräte bieten Systeme an, die eine ausreichende Qualität gewährleisten. Kontrastmittelfähige High-End-Geräte finden sich in der Stufe 3 der regelmäßig aktualisierten DEGUM-Geräteliste (http://www.degum.de/Geraetelisten.65.0.html). Vergleichende Publikationen zur Auswahl optimaler CEUS-fähiger Ultraschallgeräte fanden sich nicht.

\section{Welche Untersucherqualifikation?}

Die EFSUMB hat interdisziplinäre Empfehlungen als Qualitätsstandards für den Untersucher mit 3 unterschiedlichen Trainingsstufen formuliert $[6,7,30]$ und empfiehlt für CEUS-Untersucher den Kompetenzgrad 2 mit folgenden Voraussetzungen (EFSUMB 2010: Appendix 14):

- Erlernen von CEUS (Theorie und Praxis) unter Anleitung eines Level-3-CEUS-Trainers

- Verständnis der Technologie (Geräte und Kontrastmittel)

- Indikationen, Kontraindikationen und Artefakte

- Notfalltherapie bei Allergien

- Videoclip-Dokumentation

- Erkennung von Pathologien

- Erkennung eigener Erfahrungsgrenzen und bei Bedarf Weiterleitung an erfahrenere CEUS-Untersucher

Auch von der Deutschen Gesellschaft für Ultraschall (DEGUM) wird eine Qualitätssicherung angestrebt. Das Ziel ist, zertifizierten CEUS durch einen verpflichtenden CEUS-Kurs mit qualifizierender Abschlussprüfung flächendeckend zu etablieren. Kürzlich wurden von der DEGUM Curricula für 2 CEUS-Module entwickelt, die als Postgraduiertenkurse mit einem $50 \%$ igen Praxisanteil zertifiziert werden (www.degum.de/sektionen/ innere-medizin/kurse/module.html). CEUS-Kurse werden von
DEGUM-zertifizierten Kursleitern angeboten und u. a. von der „EUROSON SCHOOL“ (EFSUMB) durchgeführt.

\section{Möglichkeiten}

Verbesserte Detektion von Lebertumoren

In der portalvenös-sinusoidalen Phase kann leberfremdes Gewebe durch Aussparung („wash-out“) erkannt werden, da bspw. Metastasen keine leberspezifischen Gefäße aufweisen. Es konnte gezeigt werden, dass die echosignalverstärkte Sonografie mehr Metastasen detektiert als die konventionelle Sonografie [1]. Die echosignalverstärkte Sonografie der Leber ermöglicht eine mit der CT und der MRT vergleichbare Detektionsrate von Lebermetastasen. Durch die Einführung von Echosignalverstärkern konnte somit der Nachteil gegenüber den konkurrierenden kontrastverstärkten Verfahren Computertomografie (CT) und Magnetresonanztomografie (MRT) ausgeglichen werden [1, 31 - 34]. Der Einsatz der kontrastverstärkten MRT, CT und Sonografie ist dabei als ähnlich aussagekräftig zu bewerten [18]. Die Ergebnisse der ersten prospektiven europäischen Multicenterstudie (Sonovue $^{\circledR}$ ) zeigten die Machbarkeit [1]. Die DEGUM-Multicenterstudie lieferte die Bestätigung im klinischen Alltag $[3,13,14,17]$.

\section{Verbesserte Einschätzung der Dignität}

Die Einschätzung der Dignität von Lebertumoren ist im gesunden Leberparenchym bei einem Großteil der Patienten durch die echosignalverstärkte Sonografie möglich geworden. In der portalvenös-sinusoidalen Kontrastmittelphase zeigen benigne Tumoren Iso- oder Mehranreicherung und maligne Tumoren Kontrastmittelaussparung im Vergleich zum umgebenden Leberparenchym [2,35]. Die hohe Treffsicherheit gilt nicht für Patienten mit bestimmten Leberparenchymerkrankungen, da bspw. die zirrhotisch umgebaute Leber mit einem Verlust des (leberspezifischen) portalvenös-sinusoidalen Gefäßbetts einhergeht und die fortgeschrittene Fettleber zu einer schallkopfdistalen Schallschwächung führt $[10,36]$.

\section{Verbesserte Tumorcharakterisierung}

Fokal noduläre Hyperplasie und hepatozelluläres Adenom Die Differenzierung der fokal nodulären Hyperplasie und des hepatozellulären Adenoms ist durch die echosignalverstärkte Sonografie möglich geworden. Das hepatozelluläre Adenom enthält im Unterschied zur FNH keine Pfortaderäste und Gallengänge. Eine Differenzierung gelingt somit durch Analyse der portalvenösen Phase [16, 37-40].

Die FNH zeigt eine arterielle Mehranreicherung, die gerade in den ersten Sekunden sehr ausgeprägt ist ( $\bullet$ Abb. 1). Typisch ist eine zentripetale Anreicherung durch (oder bei größeren Tumoren auch mehrere) zuführende (zentral oder exzentrisch gelegene) Arterien. Die weitere Tumoranreicherung erfolgt durch eine von dieser Arterie ausgehende geordnete Gefäßarchitektur. Das hepatozelluläre Adenom ( $\bullet$ Abb. 2 ) ist in der arteriellen Phase hypervaskularisiert, allerdings ohne charakteristische Gefäßarchitektur. Es zeigt im Gegensatz zur FNH eine im Vergleich zum umgebenden Leberparenchym diskret schwächere Kontrastmittelanreicherung in den späteren Phasen, sodass eine Unterscheidung zur FNH häufig möglich ist ( $\bullet$ Tab. 1).

In den letzten Jahren wurde die Klassifikation der Leberzelladenome aufgrund histopathologischer Merkmale überarbeitet. Dadurch wurde die Variabilität dieser sowohl bildgebend als auch pathologisch differenziert zu betrachtenden sowie mitunter auch 

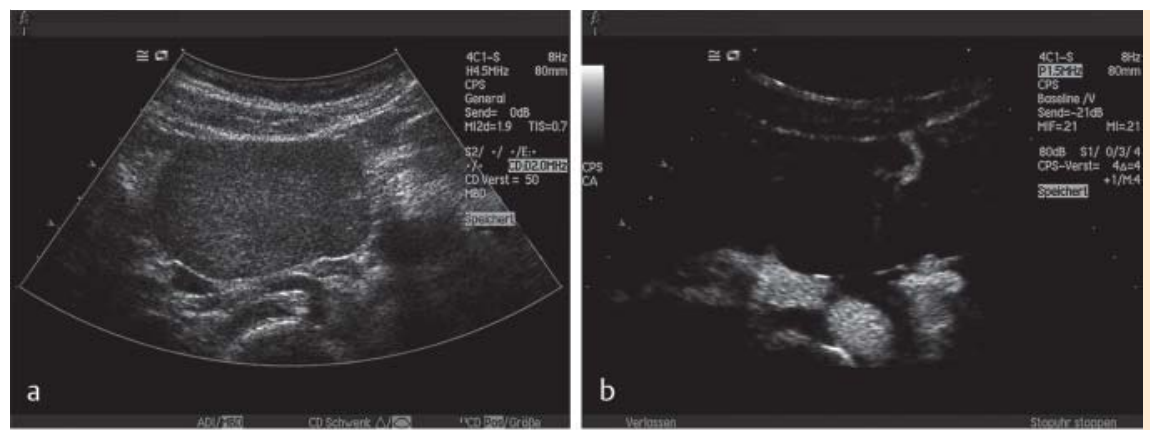

Abb. 1 Fokal noduläre Hyperplasie a. Darstellung einer fokal nodulären Hyperplasie, die zu jeder Zeit eine deutlichere Kontrastmittelanreicherung zeigt als das umgebende Leberparenchym b-g. Die Analyse der Gefäßarchitektur ist häufig erst nach einem Flash (kurzzeitige Bläschendestruktion) mit Fokussierung auf die vermutete zuführende Arterie b-e möglich. Die Narbe ist in der Spätphase darstellbar $\mathbf{g}$.
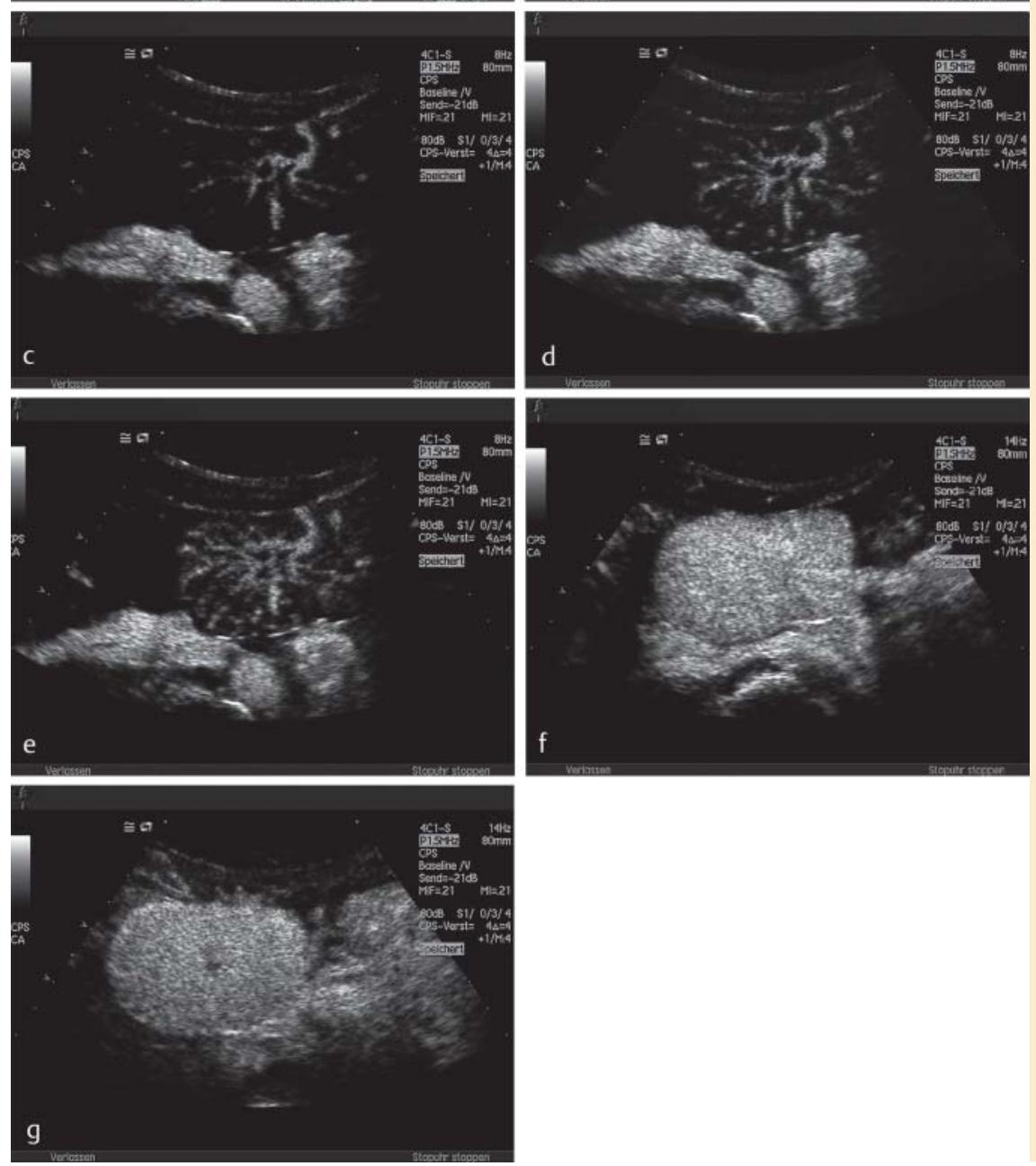

\section{$f$}
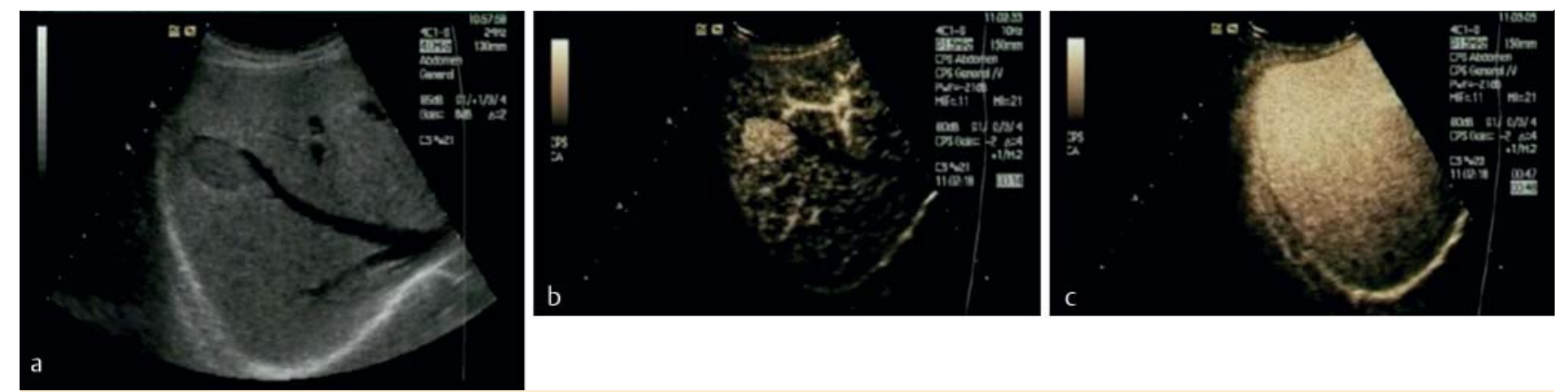

Abb.2 Hepatozelluläres Adenom im B-Bild a in der arteriellen Phase hypervaskularisiert (und somit mehranreichernd), allerdings ohne charakteristische Gefäßarchitektur b. Es zeigt im Gegensatz zur FNH eine im Vergleich zum umgebenden Leberparenchem isoechogene bis z. T. diskret schwächere Kontrastmittelanreicherung in den späteren Phasen. 
Tab. 1 Charakterisierung von 424 fokal nodulären Hyperplasien [FNH] und 36 histologisch gesicherten hepatozellulären Adenomen [HCA] [2, 12, 47].

\begin{tabular}{|c|c|c|}
\hline & FNH & HCA \\
\hline Anzahl & 424 & 36 \\
\hline Größe (mm) & $\begin{array}{l}49 \pm 24 \\
{[12-110]}\end{array}$ & $\begin{array}{l}34 \pm 22 \\
{[20-120]}\end{array}$ \\
\hline \multicolumn{3}{|l|}{ Konventionelle B-Bild-Sonografie } \\
\hline \multicolumn{3}{|l|}{ Echotextur } \\
\hline $\begin{array}{l}\text { hypoechogen (schwächer echogen als das } \\
\text { umgebende Lebergewebe) }\end{array}$ & $36^{1}$ & $8^{1}$ \\
\hline $\begin{array}{l}\text { isoechogen zum umgebenden Leberparen- } \\
\text { chym }\end{array}$ & 382 & 20 \\
\hline $\begin{array}{l}\text { stärker echogen als das umgebende Leber- } \\
\text { gewebe }\end{array}$ & 6 & 8 \\
\hline „Zentrale Narbe“ & 241 & 0 \\
\hline \multicolumn{3}{|l|}{ Farbdopplersonografie } \\
\hline hypervaskulär & 369 & 33 \\
\hline $\begin{array}{l}\text { „Radspeichenmuster“ (mit einer zentral } \\
\text { oder exzentrisch zuführenden Arterie) }\end{array}$ & 234 & 3 \\
\hline \multicolumn{3}{|l|}{ CEUS } \\
\hline $\begin{array}{l}\text { arterielle Mehranreicherung im Vergleich } \\
\text { zum umgebenden Leberparenchym }\end{array}$ & 424 & 36 \\
\hline portalvenöse Mehranreicherung & 406 & 6 \\
\hline
\end{tabular}

${ }^{1}$ Alle in einer sog. Fettleber (echoreiches Parenchym mit Arealen schwächerer Echogenität) [48].

schwierig einzuordnenden benignen Lebertumoren ganz plausibel erklärt [41, 42]. Zudem ergibt sich ein Überlappungsbereich zur FNH. Circa 15\% der FNH wurden früher als teleangiektatische FNH bezeichnet und werden heute den inflammatorischen Adenomen zugeordnet. Diese zeigen in der CEUS charakteristischerweise keine primär zentrale Radspeichenstruktur in der arteriellen Anflutung.

Es werden 3 Typen von Adenomen unterschieden, in die sich bis zu 90\% der Adenome klassifizieren lassen. In ca. 10\% werden Mischformen diskutiert bzw. ist eine Klassifikation nicht möglich [43-46].

1. Inflammatorische Adenome (früher: teleangiektatische $\mathrm{FNH}$, $40-50 \%$ aller Adenome): Klinisch bei adipösen Patienten, im B-Bild-Ultraschall oft echoarm in einer Steatose/Fettleber, in der CEUS rasche und deutliche zentripetale arterielle Kontrastmittelanflutung, in den späteren Phasen erhaltene Hypervaskularität evtl. mit zentralem wash-out.

2. Fettreiche Adenome (hepatocytic nuclear factor $\alpha-1$, HNF- $\alpha 1$ mutierte Adenome, 30 - 40\%): Klinisch bei Patienten mit Speicherkrankheiten und (langer) Kontrazeptivaeinnahme, im B-Bild echoreich, histologisch hoher Fettanteil, in der CEUS Isovaskularität oder geringe Hypervaskularität in der arteriellen Phase, Isovaskularität in der portalvenösen und sinusoidalen Phase.

3. $\beta$-Catenin-mutierte Adenome (ca. $10 \%$ ): klinisch häufiger bei Männern (androgenabhängiges Wachstum), im B-Bild echoarm, Risiko der malignen Transformation zum hepatozellulälen Karzinom: Aufgrund der Seltenheit wurden konsistente Daten zum Verhalten in der CEUS bisher nicht publiziert.

\section{Hepatozelluläre Karzinome}

Die meisten hepatozellulären Karzinome (HCC) entwickeln sich in einer zirrhotisch umgebauten Leber (90\%) und zeigen eine Mehranreicherung in der arteriellen Phase gegenüber dem umgebendem Leberparenchym (90\%); aber auch weniger vaskularisierte hepatozelluläre Karzinome werden bei ca. $10 \%$ der Patienten beobachtet [10, 36, 49-53]. Benigne Lebertumoren kommen in einer zirrhotisch umgebauten Leber ebenso häufig vor wie beim lebergesunden Patienten [51]. Allerdings ist bei Vorliegen einer Leberzirrhose und fokaler Leberläsion die Vortest-Wahrscheinlichkeit für das Vorliegen eines HCC sehr hoch. In der multizentrischen DEGUM-Studie waren bei Patienten mit Leberzirrhose HCCs 18-mal häufiger als Metastasen und 27-fach häufiger als Hämangiome [14]. Vor einer Resektion potenziell kurativ behandelbarer hepatozellulärer Karzinome sind anderweitige gutartige, insbesondere hypervaskularisierte Lebertumoren (FNH, hepatozelluläres Adenom, Shunt-Hämangiom, Metastasen insbesondere neuroendokriner Tumoren [54]) auszuschließen, um falsch indizierte Operationen zu vermeiden. Auf die wichtige und viel diskutierte Differenzialdiagnose zu intrahepatischen cholangiozellulären Karzinomen (ICC) in der zirrhotisch umgebauten Leber wird hingewiesen [10, 11, 36, 55-57). Diese Unterscheidung ist durch die arterielle Kontrastmittelanflutung vorwiegend in der Tumorperipherie sowie das deutliche frühere „Wash-out“ des ICC ( $<60 \mathrm{sec})$ in der portalen Phase im Vergleich zum HCC möglich [57]. Die deutsche S3-Leitlinie empfiehlt neben kontrastverstärkter CT oder MRT CEUS als gleichberechtigte auch primäre Bildgebungsmethode zur Charakterisierung fokaler Leberläsionen in der Leberzirrhose und damit zur Diagnosestellung des HCC [6, 7]. Die CEUS gilt auch als komplementäre zweite Bildgebung bei nicht konklusivem Befund in der primären Bildgebung durch CT oder MRT. Für die Komplettierung des lokoregionären Stagings ist zur Ausbreitungsdiagnostik in der Leber die MRT als Goldstandard empfohlen.

\section{Hämangiome}

Das aus der kontrastmittelverstärkten Computertomografie bekannte „Irisblendenphänomen“ als charakteristische Kontrastmittelanflutung im Hämangiom ist auch das typische Zeichen der echosignalverstärkten Sonografie ( $\bullet$ Abb. 3). Die periphernoduläre Kontrastmittelanreicherung wird durch die zentripetal fortschreitende (partielle) Füllung des Hämangioms ergänzt. Leider gibt es auch bei dieser typischen Kontrastmitteldarstellung Überlappungen zu Lebermetastasen gastrointestinaler Karzinome, sodass Verwechslungen möglich sind [54]. Im Unterschied zu Metastasen, die in der Spätphase gegenüber dem normalen Lebergewebe durch wash-out progredient schwächer echogen kontrastieren, imponieren Hämangiome hypervaskularisiert oder isovaskulär. Das Irisblendenphänomen kann bei Thrombosierung bzw. Fibrosierung inkomplett sein ( $\bullet$ Tab. 2, 3).

Etwa $10 \%$ der Leberhämangiome zeigen aufgrund arteriovenöser bzW. portovenöser Shunts, die in angiografischen und magnetresonanztomografischen Studien dargestellt worden sind [58], ein besonders frühes und schnelles Enhancement und werden daher als Shunt-Hämangiome oder High-flow-Hämangiome bezeichnet [59 - 62]. Da „high-flow“ nicht exakt definiert ist, sollte dieser Begriff jedoch unseres Erachtens nicht benutzt werden. ShuntHämangiome sind eher klein $(<20 \mathrm{~mm})$ und liegen typischerweise in Arealen unterschiedlicher Verfettungszonen im Vergleich zum umgebendem Lebergewebe. Das normale Lebergewebe wird vorwiegend ( $80 \%$ ) durch fett- und hormonhaltigeres Pfortaderblut durchblutet, wogegen arteriell durchblutete ShuntHämangiome eine geringere Lipid- und Insulinkonzentration als Pfortaderblut aufweisen, was zu einer geringeren Verfettung umliegender Hepatozyten führt.

\section{Anderweitige Lebertumoren}

Eine Vielzahl weiterer Lebertumoren ist zu beachten, die in der aktuellen Literatur dargestellt sind [63-67]. In der Regel ist bei 

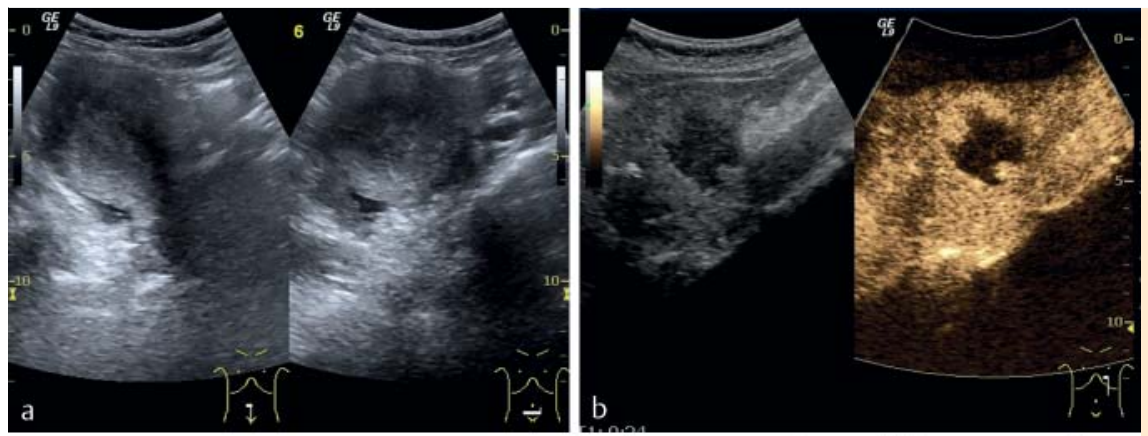

Abb. 3 Hämangiom nativ im B-Bild a 15 bis $20 \mathrm{sec}$ nach Kontrastmittelgabe $\mathbf{b}$ zentripetale Anflutung des Kontrastmittels („Irisblendenphänomen“). Die peripher noduläre Kontrastmittelanreicherung wird durch die zentripetal fortschreitende (partielle) Füllung des Hämangioms ergänzt (38 sec nach KMGabe, c). In der Spätphase (75 sec, d) imponieren Hämangiome mehr anreichernd als die Umgebung.
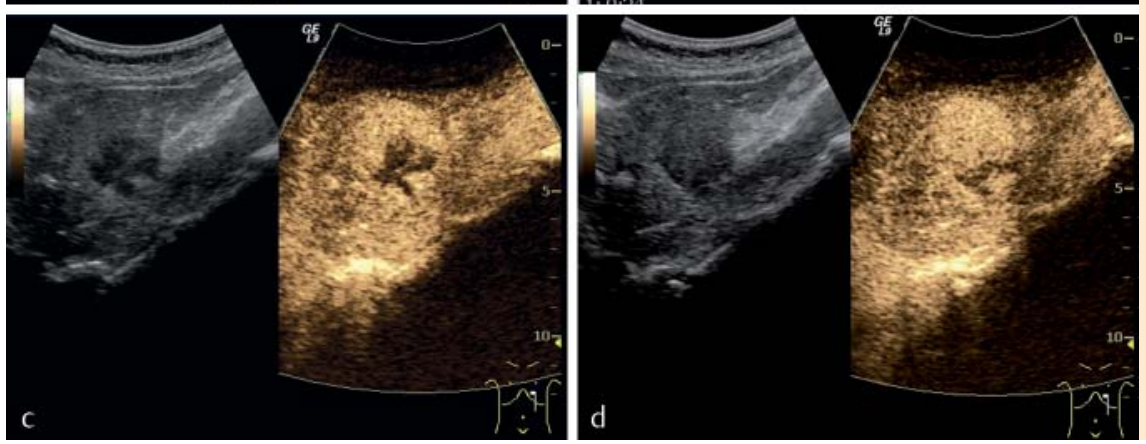

Tab. 2 Kontrastverstärkte Charakterisierung von 58 histologisch gesicherten Leberhämangiomen [58]. Die Erfahrung konnte durch mehr als 400 Kontrastmittelsonografien von Leberhämangiomen bestätigt werden [11, 55],

- Tab. 3.

\begin{tabular}{|c|c|}
\hline \multicolumn{2}{|l|}{ Charakteristika } \\
\hline \multicolumn{2}{|l|}{ arterielle Kontrastmittelanreicherung } \\
\hline peripher-noduläre Kontrastmittelanreicherung & $43 / 58(74 \%)$ \\
\hline peripheres Ringzeichen (rim-sign) & $0 / 58(0 \%)$ \\
\hline $\begin{array}{l}\text { nicht einzuordnen (bspw. aufgrund der Tumorgröße, } \\
\text { solitärer fibrotischer Knoten) }\end{array}$ & $6 / 58(10 \%)$ \\
\hline Gleichmäßige (arterielle) Anreicherung & $9 / 58(16 \%)$ \\
\hline \multicolumn{2}{|l|}{ Irisblendenphänomen (Zulaufen) } \\
\hline $\begin{array}{l}\text { vollständiges (gleichmäßiges) Irisblendenphänomen } \\
<180 \mathrm{sec}\end{array}$ & $45 / 58(78 \%)$ \\
\hline$\leq 30 \mathrm{sec}$ & $12(21 \%)$ \\
\hline$>30$ sec und $\leq 60 \mathrm{sec}$ & $6(10 \%)$ \\
\hline$>60$ sec und $\leq 180 \mathrm{sec}$ & $27(47 \%)$ \\
\hline $\begin{array}{l}\text { unvollständiges (ungleichmäßiges) Irisblenden- } \\
\text { phänomen }\end{array}$ & $13 / 58(22 \%)$ \\
\hline \multicolumn{2}{|l|}{ Sensitivität } \\
\hline peripher-noduläre Kontrastmittelanreicherung & $43 / 58(74 \%)$ \\
\hline vollständiges (gleichmäßiges) Irisblendenphänomen & $45 / 58(78 \%)$ \\
\hline Kombination beider & $57 / 58(98 \%)$ \\
\hline
\end{tabular}

unklaren Befunden die histologische Sicherung oder bei entsprechender klinischer Konsequenz die Operation angeraten.

\section{Steuerung und Verlaufskontrolle lokal ablativer Verfahren \\ $\nabla$}

Lokal ablative Verfahren spielen insbesondere eine Rolle bei der Behandlung von Lebermetastasen und von hepatozellulären Karzinomen. Mittels Hochfrequenzstrom wird eine Koagulationsnekrose über eine Ablationsnadel im Tumor erzeugt. Die Radiofrequenzthermoablation (RFA) ist neben der perkutanen Äthanolinjektion (PEI) das weltweit am häufigsten verwendete
Tab. 3 Ultraschallbefund bei 400 Patienten mit Hämangiomen, die nach den publizierten Kriterien histologisch gesicherter Hämangiome [58] mittels CEUS untersucht worden sind [12].

\begin{tabular}{|c|c|}
\hline \multicolumn{2}{|l|}{ Charakteristika } \\
\hline Anzahl & 400 \\
\hline mittlere Größe [mm] & $26 \pm 31$ \\
\hline \multicolumn{2}{|l|}{$B$-Mode } \\
\hline echoreich & $363(91 \%)$ \\
\hline isoechogen (echoärmer) & $37(9 \%)$ \\
\hline \multicolumn{2}{|l|}{ Farbdopplersonografie: Vaskularität } \\
\hline keine intraläsionalen Gefäße & $379(95 \%)$ \\
\hline intraläsionale Gefäße (hypervaskulär) & $21(5 \%)$ \\
\hline \multicolumn{2}{|l|}{ CEUS } \\
\hline $\begin{array}{l}\text { peripher noduläre Kontrastmittelanreicherung (mit Iris- } \\
\text { blendenphänomen) }\end{array}$ & $328(82 \%)$ \\
\hline $\begin{array}{l}\text { ausgeprägte gleichmäßige arterielle Kontrastmittel- } \\
\text { anreicherung }\end{array}$ & $31(8 \%)$ \\
\hline keine (oder unspezifische) Kontrastmittelanreicherung & $41(10) \%$ \\
\hline komplettes Irisblendenphänomen & $320(80 \%)$ \\
\hline inkomplettes Irisblendenphänomen & $80(20 \%)$ \\
\hline
\end{tabular}

lokal ablative Verfahren [49, 68 - 71]. Die Effektivität der RFA hinsichtlich lokaler Tumorkontrolle ist dabei etwas höher als die der PEI und hat sich somit gegenüber der PEI letztendlich durchgesetzt [72], wobei auch sehr gute PEI-Ergebnisse publiziert worden sind $(73,74]$. Auf das entsprechende Lehrbuch wird verwiesen [75].

Die echosignalverstärkte Sonografie ermöglicht die Steuerung der Nadelplatzierung insbesondere bei schlechter Visualisierung im konventionellen B-Bild und erlaubt die Ablationskontrolle. Aufgrund der hohen Ortsauflösung demarkieren sich nicht vaskularisierte nekrotische Bezirke des Tumors als ausgestanzte Defekte in sämtlichen Kontrastmittelphasen [76-79]. Damit eignet sich CEUS als ergänzendes oder alternatives Verfahren zur radiologischen Schnittbildgebung bei der Steuerung und Verlaufskontrolle der lokal ablativen Therapie maligner Lebertumoren [80 - 87]. 


\section{Einschränkungen und Fehlermöglichkeiten $\nabla$}

Fehldiagnosen bei der Kontrastmittelsonografie entstehen vorwiegend aufgrund unzureichender Kenntnisse und Erfahrungen des Untersuchers. Einzelne Gründe sind falsche Geräteeinstellung mit Bläschendestruktion bei zu hohem mechanischen Index, Unkenntnis von Artefakten sowie zu frühe Beendigung der Untersuchung vor der Spätphase [88 - 90] oder auch zu lange andauernde Beschallung aus einem Anlotungswinkel mit konsekutiver fokaler/zonaler Bläschendestruktion.

In der multizentrischen DEGUM-Studie konnten nur 6,8\% aller fokalen Leberläsionen nicht sicher durch CEUS charakterisiert werde, weitere knapp $3 \%$ der CEUS-Diagnosen erwiesen sich als falsch. In fast drei Viertel der Fälle von unklaren oder falschen CEUS-Klassifikationen fokaler Leberläsionen handelte es sich um histologisch benigne Diagnosen. Unter den 8 Fällen (0,6\%) falschnegativer CEUS-Diagnosen maligner Leberherde handelte es sich in 6 Fällen um HCCs, die als Adenome, FNH oder Hämangiom verkannt worden waren [13]. Vor jeder Kontrastmittelsonografie der Leber muss konventionell sonografisch untersucht werden, ob Zysten und Verkalkungen vorliegen, die keine Kontrastmittelanreicherung zeigen und als maligne Infiltration fehlgedeutet werden können [88-90]. Auch Nekrosen und Abszesse zeigen sowohl in der arteriellen als auch in der portalvenösen Phase keine Kontrastanflutung. Typische Tumorcharakteristika sind nur bei Raumforderungen bis $50 \mathrm{~mm}$ Größe zu erwarten, größere Tumoren zeigen unabhängig von ihrer Ätiologie degenerative Veränderungen. Auch benigne Proliferationen leberfremden Gewebes (bspw. Granulome bei Sarkoidose oder extramedulläre Blutbildungsherde bei chronischen myeloproliferativen Erkrankungen) führen aufgrund des Fehlens leberspezifischer Gefäße $\mathrm{zu}$ fokalen Minderanreicherungen in der portalvenösen und Spätphase, die sich allein bildgebend nicht von malignen Leberherden differenzieren lassen [88-91].

Eine Differenzierung des hepatozellulären Adenoms vom hepatozellulären Karzinom ist mittels bildgebender Verfahren nicht zuverlässig möglich, sodass eine histologische Untersuchung des Gewebes notwendig ist. Dabei ist zu beachten, dass selbst die histologische Beurteilung Fehlermöglichkeiten in sich birgt. Fehlerhafte histologische Einschätzungen aufgrund nicht repräsentativer Gewebeproben und Mischformen beider Tumoren wurden beobachtet, sodass im Einzelfall nur die Resektion und Gewebeaufarbeitung eindeutig ist. Das hepatozelluläre Adenom wird gehäuft bei Patienten mit Speicherkrankheiten beobachtet [92]. Eine sichere Tumorcharakterisierung von hepatozellulären Adenomen ist nur dann möglich, wenn keine sekundär regressiven Veränderungen vorliegen, wie sie besonders bei großen (>50 mm) Adenomen beobachtet werden.

Besonderheiten bei inflammatorischem Pseudotumor [93], cholangiozellulärem Adenom [5], Sarkomen [94] und anderen seltenen Tumorentitäten wurden publiziert [5]. Artefakte müssen berücksichtigt werden [89].

\section{Methodenvergleich CEUS - CT - MRT \\ $\nabla$}

Ultraschall und CEUS sind ubiquitär verfügbare schnittbildgebende Verfahren, portabel bettseitig einsetzbar und zeitlich deutlich höher auflösend als CT und MRT. Ein Vergleich der Methoden wurde kürzlich beschrieben [95, 96]. Für eine gute Darstellung im CEUS gelten die gleichen Voraussetzungen wie für den normalen Ultraschall (Luft und Knochen sowie Adipositas sind ein Untersuchungshindernis; ein qualifizierter Untersucher ist für ein gutes Ergebnis erforderlich). Darüber hinaus hat man mit CEUS deutlich weniger Kontraindikationen und Nebenwirkungen zu beachten als bei CT und MRT, wie im Folgenden aufgeführt [97-110]:

\section{US und CEUS:}

- Kontraindikationen und Nebenwirkungen:

- aktive schwere koronare Herzerkrankung, nicht stabile Angina pectoris, dekompensierte Herzinsuffizienz

- schwere pulmonale Hypertonie

- unmittelbar im Anschluss geplante extrakorporale Stoßwellenlithotripsie (ESWL)

\section{CT:}

- Kontraindikationen und Nebenwirkungen:

- Cave: Strahlenbelastung (CT Abdomen: 10 mSv = 500 Röntgen-Thorax-Aufnahmen)

- Kontrastmittel- bzw. Jodallergie (Anaphylaktischer Schock)

- Niereninsuffizienz (cave: Diabetes und Metformin)

- Hyperthyreose $(0,05 \%$ thyreotoxische Krise)

- Schwangerschaft

MRT:

- Kontraindikationen und Nebenwirkungen:

> Metall-Fremdkörper (Herzschrittmacher, Implantate, Drähte, Tätowierung, andere)

- Klaustrophobie

> Nephrotoxizität (cave Risikopatienten: Diabetes mellitus, arterielle Hypertonie)

- Nephrogene systemische Fibrose

- Anaphylaktischer Schock

- Gd-DPTA-Kontrastmittel in der Schwangerschaft Die seltene und nicht vorhersehbare anaphylaktoide (pseudoallergische) Reaktion auf Ultraschall-Kontrastmittel entspricht der anaphylaktoiden Reaktion auf andere lipid(liposomal)umhüllte Therapeutika (C activation-related pseudoallergy, CARPA). Dieser anaphylaktoiden (pseudoallergischen) Reaktion liegt eine Komplementaktivierung zugrunde [110 - 113]. Dabei treten typischerweise Engegefühl in Brust und Hals sowie arterielle Hypotonie, Tachykardie und andere auf eine Allergie weisende Symptome auf. Der (präventive) Einsatz von Glukokortikoiden und Antihistaminika wird empfohlen. Die Therapie entspricht der des anaphylaktischen Schocks.

\section{Weitere Techniken und Zukunftsperspektiven $\nabla$}

Die kontrastverstärkte 3D-Sonografie wurde beschrieben, ist aber nicht etabliert [114]. Die echosignalverstärkte Sonografie ist ebenfalls bei der Tumorcharakterisierung und Abgrenzung gegenüber entzündlichen Veränderungen hilfreich [115]. Fusionstechniken zwischen CEUS und radiologischer Schnittbildgebung sind insbesondere für die Steuerung von Interventionen hilfreich [116-118].

Zur Objektivierung der Kontrastmittelanreicherung sind mit wissenschaftlicher Zielsetzung Studien zur Quantifizierung (dynamic contrast enhanced ultrasound, DCE-US) mit Auswertung der Kontrastmittelkinetiken (TIC, time intensity curves) publiziert worden [119]. Zunächst hat sich diese Technik noch nicht in der klinischen Anwendung durchsetzen können [120]. Dies ist u.a. bedingt durch die fehlende Standardisierung, die Notwendigkeit 
einer aufwendigen Nachbearbeitung sowie die fehlende Definition von Bereichen, in denen diese Techniken gegenüber dem konventionellen Vorgehen Vorteile bieten (z. B. frühzeitige Analyse des therapeutischen Ansprechens von Tumoren) [36, 121, 122]. Insgesamt betrachtet ist die Entwicklung dieser Technik sinnvoll und wird eine objektivere Evaluierung der Kontrastmittelkinetik ermöglichen [38]. Leitlinien wurden publiziert [8].

\section{Zusammenfassung}

Von den derzeitigen Kontrastmitteln zur Leber-Kontrastmittelsonografie gilt SonoVue ${ }^{\circledR}$ in Europa aufgrund der Zulassungscharakteristika und publizierten Evidenz als Echosignalverstärker der ersten Wahl.

Bei der Geräteauswahl ist auf höchste Qualität zu achten, d. h. ein High-End-Gerät der Stufe 3 in der regelmäßig aktualisierten DEGUM-Geräteliste.

Wer Kontrastmittelsonografie der Leber durchführt, muss Indikationen, Kontraindikationen und Artefakte kennen, die Notfalltherapie und die Videoclip-Dokumentation beherrschen und sollte nach EFSUMB-Empfehlung diese Methode unter Anleitung eines Level-3-CEUS-Trainers erlernt haben.

Die CEUS der Leber ermöglicht gegenüber der B-Bild-Sonografie eine deutlich verbesserte, mit der CT und der MRT vergleichbare Detektionsrate von Lebermetastasen. Auch die Einschätzung der Dignität und der Artdiagnose von Lebertumoren (Hämangiom und $\mathrm{FNH}$ ) ist im gesunden Leberparenchym bei einem Großteil der Patienten möglich.

Für das HCC ist CEUS in Deutschland neben kontrastverstärkter CT und MRT als alternative kontrastgestützte Bildgebungsmethode in der aktuellen S3-Leitlinie empfohlen und etabliert. Dies gilt ähnlich für die EFSUMB-Leitlinien sowie bspw. in Italien und Japan.

CEUS ermöglicht bei der Radiofrequenzablation die Ablationskontrolle durch Demarkierung nekrotischer Tumoranteile.

Fehlermöglichkeiten entstehen insbesondere bei falscher Geräteeinstellung oder Bedienung (zu hoher mechanischer Index mit Bläschenzerstörung und Schallabschwächung) sowie bei vorzeitiger Beendigung der Untersuchung. Mögliche Durchblutungsänderungen in Geweben infolge von Degenerations- und Schrumpfungsprozessen in Tumoren müssen dem Untersucher bekannt sein, ebenso wie Kontraindikationen der Methode (bekannte pseudoallergische Reaktion in der Anamnese, Irrelevanz der Befunderhebung bei akut bedrohlicher kardiovaskulärer Krankheit sowie vor geplanter ESWL).

\section{Literatur}

1 Dietrich CF, Kratzer W, Strobel D et al. Assessment of metastatic liver disease in patients with primary extrahepatic tumors by contrast-enhanced sonography versus CT and MRI. World J Gastroenterol 2006; 12: $1699-1705$

2 Dietrich CF, Ignee A, Trojan J et al. Improved characterisation of histologically proven liver tumours by contrast enhanced ultrasonography during the portal venous and specific late phase of SHU 508A. Gut 2004; 53: $401-405$

3 Strobel D, Seitz K, Blank W et al. Contrast-enhanced ultrasound for the characterization of focal liver lesions-diagnostic accuracy in clinical practice (DEGUM multicenter trial). Ultraschall in Med 2008; 29: 499-505

4 Claudon $M$, Cosgrove D, Albrecht $T$ et al. Guidelines and good clinical practice recommendations for contrast enhanced ultrasound (CEUS) - update 2008. Ultraschall in Med 2008; 29: 28-44

5 Piscaglia F, Nolsoe C, Dietrich CF et al. The EFSUMB Guidelines and Recommendations on the Clinical Practice of Contrast Enhanced Ultra- sound (CEUS): update 2011 on non-hepatic applications. Ultraschall in Med 2012; 33: $33-59$

6 Claudon M, Dietrich CF, Choi BI et al. Guidelines and good clinical practice recommendations for contrast enhanced ultrasound (CEUS) in the liver-update 2012: a WFUMB-EFSUMB initiative in cooperation with representatives of AFSUMB, AIUM, ASUM, FLAUS and ICUS. Ultraschall in Med 2013; 34: 11 - 29

7 Claudon M, Dietrich CF, Choi BI et al. Guidelines and good clinical practice recommendations for Contrast Enhanced Ultrasound (CEUS) in the liver - update 2012: AWFUMB-EFSUMB initiative in cooperation with representatives of AFSUMB, AIUM, ASUM, FLAUS and ICUS. Ultrasound Med Biol 2013; 39: $187-210$

8 Dietrich CF, Averkiou MA, Correas JM et al. An EFSUMB introduction into Dynamic Contrast-Enhanced Ultrasound (DCE-US) for quantification of tumour perfusion. Ultraschall in Med 2012; 33: $344-351$

9 Dietrich CF, Cui XW, Boozari B et al. Contrast-enhanced ultrasound (CEUS) in the diagnostic algorithm of hepatocellular and cholangiocellular carcinoma, comments on the AASLD guidelines. Ultraschall in Med 2012; 33: S57-S66

10 Barreiros AP, Piscaglia F, Dietrich CF. Contrast enhanced ultrasound for the diagnosis of hepatocellular carcinoma (HCC): comments on AASLD guidelines. J Hepatol 2012; 57: 930 - 932

11 Dietrich CF, Cui XW, Schreiber-Dietrich DG et al. EFSUMB guidelines 2011: comments and illustrations. Ultraschall in Med 2012; 33: S11 - S21

12 Dietrich CF. Liver tumor characterization-comments and illustrations regarding guidelines. Ultraschall in Med 2012; 33: S22 - S30

13 Bernatik T, Seitz K, Blank W et al. Unclear focal liver lesions in contrastenhanced ultrasonography-lessons to be learned from the DEGUM multicenter study for the characterization of liver tumors. Ultraschall in Med 2010; 31: $577-581$

14 Seitz K, Greis C, Schuler A et al. Frequency of tumor entities among liver tumors of unclear etiology initially detected by sonography in the noncirrhotic or cirrhotic livers of 1349 patients. Results of the DEGUM multicenter study. Ultraschall in Med 2011; 32: 598-603

15 Seitz K, Strobel D, Bernatik $T$ et al. Contrast-Enhanced Ultrasound (CEUS) for the characterization of focal liver lesions - prospective comparison in clinical practice: CEUS vs. CT (DEGUM multicenter trial). Parts of this manuscript were presented at the Ultrasound Dreilandertreffen 2008, Davos. Ultraschall in Med 2009; 30: 383 - 389

16 Strobel D, Seitz K, Blank W et al. Tumor-specific vascularization pattern of liver metastasis, hepatocellular carcinoma, hemangioma and focal nodular hyperplasia in the differential diagnosis of 1349 liver lesions in contrast-enhanced ultrasound (CEUS). Ultraschall in Med 2009; 30: 376-382

17 Strobel D, Bernatik T, Blank W et al. Diagnostic accuracy of CEUS in the differential diagnosis of small $(</=20 \mathrm{~mm})$ and subcentimetric $(</=$ $10 \mathrm{~mm}$ ) focal liver lesions in comparison with histology. Results of the DEGUM multicenter trial. Ultraschall in Med 2011; 32: $593-597$

18 Westwood M, Joore M, Grutters J et al. Contrast-enhanced ultrasound using SonoVue(R) (sulphur hexafluoride microbubbles) compared with contrast-enhanced computed tomography and contrast-enhanced magnetic resonance imaging for the characterisation of focal liver lesions and detection of liver metastases: a systematic review and cost-effectiveness analysis. Health Technol Assess 2013; 17 (16): 1 - 243

19 Lorusso A, Quaia E, Poillucci G et al. Activity-based cost analysis of contrast-enhanced ultrasonography (CEUS) related to the diagnostic impact in focal liver lesion characterisation. Insights Imaging 2015, [epub ahead of print]

20 Dietrich CF, Riemer-Hommel P. Challenges for the German Health Care System. Z Gastroenterol 2012; 50: $557-572$

21 Dietrich CF, Mäurer M, Riemer-Hommel P. Challenges for the German Health Care System. Pharmaceuticals. Endo heute 2014; 27: 45-53

22 Dietrich CF. Editorial on the contribution "Challenges for the German Health Care System". Z Gastroenterol 2012; 50: 555 - 556

23 Schreiber-Dietrich D, Dietrich CF. Contrast enhanced ultrasound (CEUS) and off-label use (in children). Ultraschall in Med 2012; 33: 295-296

24 Schreiber-Dietrich DG, Cui XW, Piscaglia F et al. Contrast enhanced ultrasound in pediatric patients: a real challenge. Z Gastroenterol 2014; 52 (10): $1178-1184$

25 Albrecht T, Blomley M, Bolondi L et al. Guidelines for the use of contrast agents in ultrasound. Ultraschall in Med 2004; 25: 249-256

26 Dietrich CF, Schreiber-Dietrich D, Schuessler G et al. Contrast enhanced ultrasound of the liver-state of the art. Dtsch Med Wochenschr 2007; 132 (22): 1225 - 1231

27 Dietrich CF. Characterisation of focal liver lesions with contrast enhanced ultrasonography. Eur J Radiol 2004; 51 Suppl: S9-S17 
28 von Herbay A, Haeussinger D, Gregor $M$ et al. Characterization and detection of hepatocellular carcinoma (HCC): comparison of the ultrasound contrast agents SonoVue (BR 1) and Levovist (SH U 508A). Ultraschall in Med 2007; 28: 168-175

29 Sugimoto K, Moriyasu F, Saito K et al. Comparison of Kupffer-phase Sonazoid-enhanced sonography and hepatobiliary-phase gadoxetic acidenhanced magnetic resonance imaging of hepatocellular carcinoma and correlation with histologic grading. J Ultrasound Med 2012; 31: $529-538$

30 Dietrich CF, Rudd L. The EFSUMB website, a guide for better understanding. Med Ultrason 2013; 15: 215 - 223

31 Bernatik T, Becker D, Neureiter D et al. Detection of liver metastasescomparison of contrast-enhanced ultrasound using first versus second generation contrast agents. Ultraschall in Med 2003; 24: 175-179

32 Bernatik T, Strobel D, Hahn EG et al. Detection of liver metastases: comparison of contrast-enhanced wide-band harmonic imaging with conventional ultrasonography. J Ultrasound Med 2001; 20: 509-515

33 Oldenburg A, Hohmann J, Foert E et al. Detection of hepatic metastases with low MI real time contrast enhanced sonography and SonoVue. Ultraschall in Med 2005; 26: $277-284$

34 Albrecht T, Blomley MJ, Burns PN et al. Improved detection of hepatic metastases with pulse-inversion US during the liver-specific phase of SHU 508A: multicenter study. Radiology 2003; 227: 361 - 370

35 von Herbay A, Vogt C, Haussinger D. Late-phase pulse-inversion sonography using the contrast agent levovist: differentiation between benign and malignant focal lesions of the liver. Am J Roentgenol Am J Roentgenol 2002; 179: 1273-1279

36 Piscaglia F, Cucchetti A, Dietrich CF et al. Towards new tools for refined management of patients with advanced hepatocellular carcinoma under systemic therapy: some enthusiasm with a word of caution. J Hepatol 2013; 59: $924-925$

37 Dietrich CF, Schuessler G, Trojan J et al. Differentiation of focal nodular hyperplasia and hepatocellular adenoma by contrast-enhanced ultrasound. Br J Radiol 2005; 78 (932): $704-707$

38 Cui XW, Ignee A, Jedrzejczyk M et al. Dynamic Vascular Pattern (DVP), a quantification tool for contrast enhanced ultrasound. Z Gastroenterol 2013: 51: 427-431

39 Wilson SR, Burns PN. An algorithm for the diagnosis of focal liver masses using microbubble contrast-enhanced pulse-inversion sonography. Am J Roentgenol Am J Roentgenol 2006; 186: 1401 - 1412

40 Piscaglia F, Venturi A, Mancini $M$ et al. Diagnostic features of real-time contrast-enhanced ultrasound in focal nodular hyperplasia of the liver. Ultraschall in Med 2010; 31: 276-282

41 Bosman FT, Carneiro F, Hruban RH et al. WHO Classification of Tumours of the Digestive System. 4ed. World Health Organisation; 2010

42 Shanbhogue A, Shah SN, Zaheer A et al. Hepatocellular adenomas: current update on genetics, taxonomy, and management. J Comput Assist Tomogr 2011; 35: 159-166

43 Bioulac-Sage P, Rebouissou S, Thomas C et al. Hepatocellular adenoma subtype classification using molecular markers and immunohistochemistry. Hepatology 2007; 46: $740-748$

44 Balabaud C, Al Rabih WR, Chen PJ et al. Focal Nodular Hyperplasia and Hepatocellular Adenoma around the World Viewed through the Scope of the Immunopathological Classification. Int J Hepatol 2013; 2013: 268625

45 Laumonier H, Cailliez H, Balabaud C et al. Role of contrast-enhanced sonography in differentiation of subtypes of hepatocellular adenoma: correlation with MRI findings. Am J Roentgenol Am J Roentgenol 2012; 199: $341-348$

46 Manichon AF, Bancel B, Durieux-Millon M et al. Hepatocellular adenoma: evaluation with contrast-enhanced ultrasound and MRI and correlation with pathologic and phenotypic classification in 26 lesions. HPB Surg 2012; 2012: 418745

47 Dietrich CF, Schuessler G, Trojan J et al. Differentiation of focal nodular hyperplasia and hepatocellular adenoma by contrast-enhanced ultrasound. Br J Radiol 2005; 78 (932): $704-707$

48 Hirche TO, Ignee A, Hirche $\mathrm{H}$ et al. Evaluation of hepatic steatosis by ultrasound in patients with chronic hepatitis $C$ virus infection. Liver Int 2007; 27: $748-757$

49 Strobel D, Kleinecke C, Hansler J et al. Contrast-enhanced sonography for the characterisation of hepatocellular carcinomas-correlation with histological differentiation. Ultraschall in Med 2005; 26: 270 - 276

50 Bolondi L, Gaiani S, Celli $N$ et al. Characterization of small nodules in cirrhosis by assessment of vascularity: the problem of hypovascular hepatocellular carcinoma. Hepatology 2005; 42: 27 - 34
51 Ignee A, Weiper D, Schuessler G et al. Sonographic characterisation of hepatocellular carcinoma at time of diagnosis. Z Gastroenterol 2005; 43: $289-294$

52 Gaiani S, Celli N, Piscaglia F et al. Usefulness of contrast-enhanced perfusional sonography in the assessment of hepatocellular carcinoma hypervascular at spiral computed tomography. J Hepatol 2004; 41: $421-426$

53 Bolondi L. Screening for hepatocellular carcinoma in cirrhosis. J Hepatol 2003; 39: $1076-1084$

54 Mork H, Ignee A, Schuessler G et al. Analysis of neuroendocrine tumour metastases in the liver using contrast enhanced ultrasonography. Scand J Gastroenterol 2007; 42: 652-662

55 Dietrich CF, Maddalena ME, Cui XW et al. Liver tumor characterizationreview of the literature. Ultraschall in Med 2012; 33 Suppl 1: S3 - 10

56 Wildner D, Pfeifer L, Goertz RS et al. Dynamic contrast-enhanced ultrasound (DCE-US) for the characterization of hepatocellular carcinoma and cholangiocellular carcinoma. Ultraschall in Med 2014; 35: 522 - 527

57 Wildner D, Bernatik T, Greis C et al. CEUS in Hepatocellular Carcinoma and Intrahepatic Cholangiocellular Carcinoma in 320 Patients - Early or Late Washout Matters: A Subanalysis of the DEGUM Multicenter Trial. Ultraschall in Med 2015; 36: $132-139$

58 Dietrich CF, Mertens JC, Braden B et al. Contrast-enhanced ultrasound of histologically proven liver hemangiomas. Hepatology 2007; 45: 1139 - 1145

59 Morita $Y$, Shirado $H$, Shinohara $M$ et al. Arterial-portal shunt in cavernous hemangioma of the liver. Rinsho Hoshasen 1983; 28 (12): 1507 1510

60 Morita M, Sakakiyama Y, Shiono H et al. An autopsy case of a cavernous hemangioma with solitary fibromatous fibrosis within the cavernous space in the liver of an adult. Sapporo Igaku Zasshi 1970; 38: 1-7

61 Girard C, Graham JH, Johnson WC. Arteriovenous hemangioma (arteriovenous shunt). A clinicopathological and histochemical study. J Cutan Pathol 1974; 1: 73-87

62 Mostbeck A, Grunert V, Valencak E. Angioscintigraphy and shunt volume determination in a.-v. hemangiomas. Wien Med Wochenschr 1970; 120 (47): $872-875$

63 Schuessler G, Ignee A, Hirche T et al. Improved detection and characterisation of liver tumors with echo-enhanced ultrasound. Z Gastroenterol 2003; 41: 1167-1176

64 Rickes $S$, Wermke T, Ocran K et al. Contrast behaviour of a angiomyolipoma of the liver at echo-enhanced power-Doppler sonography. Ultraschall in Med 2002; 23: 338 - 340

65 Rickes S, Ocran K, Schulze S et al. Evaluation of Doppler sonographic criteria for the differentiation of hepatocellular carcinomas and regenerative nodules in patients with liver cirrhosis. Ultraschall in Med 2002; $23: 83-90$

66 Rickes S, Ocran KW, Gerstenhauer G et al. Evaluation of diagnostic criteria for liver metastases of adenocarcinomas and neuroendocrine tumours at conventional ultrasound, unenhanced power Doppler sonography and echo-enhanced ultrasound. Dig Dis 2004; 22: 81 - 86

67 Bauditz J, Rudolph B, Wermke W. Osteoclast-like giant cell tumors of the pancreas and liver. World J Gastroenterol 2006; 12: $7878-7883$

68 Hansler J, Witte A, Strobel D et al. Radio-frequency-ablation (RFA) with wet electrodes in the treatment of primary and secondary liver tumours. Ultraschall in Med 2003; 24: 27 - 33

69 Hansler J, Neureiter D, Wasserburger $M$ et al. Percutaneous US-guided radiofrequency ablation with perfused needle applicators: improved survival with the VX2 tumor model in rabbits. Radiology 2004; 230 : $169-174$

70 Frieser $M$, Schaber $S$, Peters A et al. Radiofrequency ablation using perfused needle electrodes - study of intermittent and continuous triple needle application ex vivo. Eur Surg Res 2005; 37: 312-316

71 Hansler J, Wissniowski TT, Schuppan D et al. Activation and dramatically increased cytolytic activity of tumor specific T lymphocytes after radiofrequency ablation in patients with hepatocellular carcinoma and colorectal liver metastases. World J Gastroenterol 2006; 12: 3716-3721

72 Lencioni R, Cioni D, Crocetti L et al. Early-stage hepatocellular carcinoma in patients with cirrhosis: long-term results of percutaneous imageguided radiofrequency ablation. Radiology 2005; 234: 961 -967

73 Plentz RR, Lankisch TO, Basturk M et al. Prospective analysis of German patients with hepatocellular carcinoma undergoing transcatheter arterial chemoembolization with or without prophylactic antibiotic therapy. J Gastroenterol Hepatol 2005; 20: 1134-1136

74 Dettmer A, Kirchhoff TD, Gebel M et al. Combination of repeated singlesession percutaneous ethanol injection and transarterial chemoembo- 
lisation compared to repeated single-session percutaneous ethanol injection in patients with non-resectable hepatocellular carcinoma. World J Gastroenterol 2006; 12: 3707-3715

75 Dietrich CF, Nuernberg D. Interventioneller Ultraschall. Lehrbuch und Atlas für die Interventionelle Sonografie. Stuttgart, New York: Thieme; 2011

76 Goldberg SN, Solbiati L. Tumor dissemination after radiofrequency ablation of hepatocellular carcinoma. Hepatology 2001; 34: 609-1

77 Solbiati L, Ierace T, Tonolini $M$ et al. Radiofrequency thermal ablation of hepatic metastases. Eur J Ultrasound 2001; 13: 149-158

78 Solbiati L, Ierace T, Tonolini $M$ et al. Guidance and monitoring of radiofrequency liver tumor ablation with contrast-enhanced ultrasound. Eur J Radiol 2004; 51: S19-S23

79 Livraghi T, Solbiati L, Meloni F et al. Percutaneous radiofrequency ablation of liver metastases in potential candidates for resection: the "testof-time approach". Cancer 2003; 97: 3027-3035

80 Frieser M, Kiesel J, Lindner A et al. Efficacy of contrast-enhanced US versus CT or MRI for the therapeutic control of percutaneous radiofrequency ablation in the case of hepatic malignancies. Ultraschall in Med 2011; 32: $148-153$

81 Dong Y, Wang WP, Gan YH et al. Radiofrequency ablation guided by contrast-enhanced ultrasound for hepatic malignancies: preliminary results. Clin Radiol 2014; 69: 1129-1135

82 Mauri G, Porazzi E, Cova L et al. Intraprocedural contrast-enhanced ultrasound (CEUS) in liver percutaneous radiofrequency ablation: clinical impact and health technology assessment. Insights Imaging 2014; 5: 209-216

83 Minami Y, Nishida N, Kudo M. Therapeutic response assessment of RFA for HCC: contrast-enhanced US, CT and MRI. World J Gastroenterol 2014; 20: 4160-4166

84 Inoue T, Kudo M, Hatanaka K et al. Usefulness of contrast-enhanced ultrasonography to evaluate the post-treatment responses of radiofrequency ablation for hepatocellular carcinoma: comparison with dynamic CT. Oncology 2013; 84: 51-57

85 Shiozawa K, Watanabe M, Takayama $R$ et al. Evaluation of local recurrence after treatment for hepatocellular carcinoma by contrast-enhanced ultrasonography using Sonazoid: comparison with dynamic computed tomography. J Clin Ultrasound 2010; 38: $182-189$

$86 \mathrm{Du}$ J, Li HL, Zhai B et al. Radiofrequency Ablation for Hepatocellular Carcinoma: Utility of Conventional Ultrasound and Contrast-enhanced Ultrasound in Guiding and Assessing Early Therapeutic Response and Short-term Follow-up Results. Ultrasound Med Biol 2015, [epub ahead of print]

87 Catalano O, Izzo F, Vallone P et al. Integrating contrast-enhanced sonography in the follow-up algorithm of hepatocellular carcinoma treated with radiofrequency ablation: single cancer center experience. Acta Radiol 2015; 56: $133-142$

88 Dietrich CF, Ignee A, Greis C et al. Artifacts and pitfalls in contrast-enhanced ultrasound of the liver. Ultraschall in Med 2014; 35: 108-125

89 Dietrich $C F$, Ignee A, Hocke $M$ et al. Pitfalls and artefacts using contrast enhanced ultrasound. Z Gastroenterol 2011; 49: 350-356

90 Cui XW, Ignee A, Hocke $M$ et al. Prolonged Heterogeneous Liver Enhancement on Contrast-Enhanced Ultrasound. Ultraschall in Med 2014; 35: $246-252$

91 Goertz RS, Janka R, Nagel A et al. Eosinophilic infiltration of the liver and pancreas mimicking metastatic disease. Z Gastroenterol 2010; 48: $1138-1140$

92 Gossmann J, Scheuermann EH, Frilling A et al. Multiple adenomas and hepatocellular carcinoma in a renal transplant patient with glycogen storage disease type 1a (von Gierke disease). Transplantation 2001; 72: $343-344$

93 Schuessler G, Fellbaum C, Fauth F et al. The Inflammatory Pseudotumour - an Unusual Liver Tumour. Ultraschall in Med 2006; 27: 273-279

94 Trojan J, Hammerstingl R, Engels $K$ et al. Contrast-enhanced ultrasound in the diagnosis of malignant mesenchymal liver tumors. J Clin Ultrasound 2010; 38: 227-231

95 Chiorean L, Cantisani V, Jenssen C et al. Focal masses in a non-cirrhotic liver: The additional benefit of CEUS over baseline imaging. Eur J Radiol 2015, [epub ahead of print]

96 Chiorean L, Caraiani C, Radzina $M$ et al. Vascular phases in imaging and their role in focal liver lesions assessment. J Clin Hemorheol Microcirculation 2015, in press.

97 Brenner DJ, Doll R, Goodhead DT et al. Cancer risks attributable to low doses of ionizing radiation: assessing what we really know. Proc Natl Acad Sci U S A 2003; 100: 13761 - 13766
98 Hall EJ, Brenner DJ. Cancer risks from diagnostic radiology. Br J Radiol 2008; 81: $362-378$

99 Brenner DJ, Hall EJ. Computed tomography-an increasing source of radiation exposure. N Engl J Med 2007; 357: 2277-2284

100 Hricak H, Brenner DJ, Adelstein SJ et al. Managing radiation use in medical imaging: a multifaceted challenge. Radiology 2011; 258: 889905

101 Stacul F, van der Molen AJ, Reimer P et al. Contrast induced nephropathy: updated ESUR Contrast Media Safety Committee guidelines. Eur Radiol 2011; 21: 2527-2541

102 Morcos SK, Thomsen HS, Exley CM. Contrast media: interactions with other drugs and clinical tests. Eur Radiol 2005; 15: 1463-1468

103 van der Molen AJ, Thomsen HS, Morcos SK. Effect of iodinated contrast media on thyroid function in adults. Eur Radiol 2004; 14: 902-907

104 Thomsen HS, Morcos SK. ESUR guidelines on contrast media. Abdom Imaging 2006; 31: $131-140$

105 Bellin MF, Stacul F, Webb JA et al. Late adverse reactions to intravascular iodine based contrast media: an update. Eur Radiol 2011; 21: $2305-2310$

106 Morcos SK, Bellin MF, Thomsen HS et al. Reducing the risk of iodine-based and MRI contrast media administration: recommendation for a questionnaire at the time of booking. Eur J Radiol 2008; 66: 225-229

107 Bellin MF, Webb JA, van der Molen AJ et al. Safety of MR liver specific contrast media. Eur Radiol 2005; 15: $1607-1614$

108 Jakobsen JA, Oyen R, Thomsen HS et al. Safety of ultrasound contrast agents. Eur Radiol 2005; 15: 941 -945

109 Thomsen HS, Morcos SK, Almen T et al. Nephrogenic systemic fibrosis and gadolinium-based contrast media: updated ESUR Contrast Medium Safety Committee guidelines. Eur Radiol 2013; 23: 307-318

110 Piscaglia F, Bolondi L. The safety of Sonovue in abdominal applications: retrospective analysis of 23188 investigations. Ultrasound Med Biol 2006; 32: 1369-1375

111 Szebeni J, Muggia F, Gabizon A et al. Activation of complement by therapeutic liposomes and other lipid excipient-based therapeutic products: prediction and prevention. Adv Drug Deliv Rev 2011; 63: $1020-1030$

112 Szebeni J, Baranyi L, Savay S et al. Role of complement activation in hypersensitivity reactions to doxil and hynic PEG liposomes: experimental and clinical studies. J Liposome Res 2002; 12: 165-172

113 Kitzman DW, Goldman ME, Gillam LD et al. Efficacy and safety of the novel ultrasound contrast agent perflutren (definity) in patients with suboptimal baseline left ventricular echocardiographic images. Am J Cardiol 2000; 86: 669-674

114 Dietrich CF. 3D real time contrast enhanced ultrasonography, a new technique. Rofo 2002; 174: $160-163$

115 Hocke M, Schulze E, Gottschalk P et al. Contrast-enhanced endoscopic ultrasound in discrimination between focal pancreatitis and pancreatic cancer. World J Gastroenterol 2006; 12: 246-250

116 Min JH, Lim HK, Lim S et al. Radiofrequency ablation of very-early-stage hepatocellular carcinoma inconspicuous on fusion imaging with B-mode US: value of fusion imaging with contrast-enhanced US. Clin Mol Hepatol 2014; 20: 61 - 70

117 Minami T, Minami Y, Chishina $H$ et al. Combination guidance of contrast-enhanced US and fusion imaging in radiofrequency ablation for hepatocellular carcinoma with poor conspicuity on contrast-enhanced US/fusion imaging. Oncology 2014; 87: 55-62

118 Lee MW, Rhim H, Cha DI et al. Planning US for percutaneous radiofrequency ablation of small hepatocellular carcinomas $(1-3 \mathrm{~cm})$ : value of fusion imaging with conventional US and CT/MR images. J Vasc Interv Radiol 2013; 24: 958-965

119 Frohlich E, Muller R, Cui XW et al. Dynamic contrast-enhanced ultrasound for quantification of tissue perfusion. J Ultrasound Med 2015; 34: $179-196$

120 Ignee A, Jedrejczyk M, Schuessler G et al. Quantitative contrast enhanced ultrasound of the liver for time intensity curves-Reliability and potential sources of errors. Eur J Radiol 2010; 73: 153-158

121 Zocco MA, Garcovich M, Lupascu A et al. Early prediction of response to sorafenib in patients with advanced hepatocellular carcinoma: the role of dynamic contrast enhanced ultrasound. J Hepatol 2013; 59: $1014-1021$

122 Knieling F, Waldner MJ, Goertz RS et al. Early response to anti-tumoral treatment in hepatocellular carcinoma-can quantitative contrast-enhanced ultrasound predict outcome? Ultraschall in Med 2013; 34: $38-46$ 